\title{
Anabases
}

ANABASES Traditions et réceptions de l'Antiquité

12 | 2010

Varia

\section{Sarx, Hiems}

\section{Catherine Soulier}

\section{OpenEdition}

Journals

Édition électronique

URL : http://journals.openedition.org/anabases/1238

DOI : 10.4000/anabases.1238

ISSN : 2256-9421

\section{Éditeur}

E.R.A.S.M.E.

\section{Édition imprimée}

Date de publication : 1 octobre 2010

Pagination : 220-225

ISSN : 1774-4296

\section{Référence électronique}

Catherine Soulier, «Sarx, Hiems », Anabases [En ligne], 12 | 2010, mis en ligne le 01 octobre 2013, consulté le 21 octobre 2019. URL : http://journals.openedition.org/anabases/1238 ; DOI : 10.4000/ anabases. 1238

Ce document a été généré automatiquement le 21 octobre 2019

(c) Anabases 


\title{
Sarx, Hiems
}

\author{
Catherine Soulier
}

Je ne lis que le français,

c'est-à-dire, aussi,

le latin et le grec. [...]

Tradition, traduction où

cette langue sans cesse

est reconduite, traduite

en elle-même.

1 En 1977, l'année où il commence les Petits Traités, textes fragmentaires, rebelles et d'une bien étrange érudition, Pascal Quignard donne deux minces livres que leur date de publication place au nombre de ceux qu'il englobera plus tard, le temps sans doute d'une de ces déclarations imprudentes favorisées par le rituel de l'entretien, dans la catégorie des « exercices d'arpège les plus saugrenus », fruits de son entraînement dans « les mouvements underground de l'après $68^{1}$ ». L'un, Sarx, est publié aux éditions d'Aimé Maeght avec, à la demande de Jacques Dupin, des dessins de Gérard Titus-Carmel. L'autre, Hiems, paraît aux éditions Orange Export Ltd., fondées quelques années auparavant par Emmanuel Hocquard et Raquel Levy².

2 Sarx, Hiems : deux livres et deux mots.

3 Les livres : forme donnée à deux textes de genre indécis, troués de vastes blancs; entre essai, pensées, poème. En somme des Petits traités avant la lettre, qu'il suffirait d'un sous-titre : « Sur le sarcasme » (ou « Sur la tête écorchée »), « Sur l'hiver de la parole »... pour inscrire dans l'ensemble à venir.

4 Les mots : deux substantifs monosyllabiques, l'un grec, l'autre latin.

5 Ces deux mots, donc, tirent après eux deux langues. Deux langues de culture, deux langues savantes; celles des humanités classiques, dont la présence insistante, désormais mesurable dans une œuvre qui s'est considérablement accrue, vaut à Pascal Quignard le qualificatif d'érudit, qu'il a si souvent récusé.

6 Langues mortes, dit-on, pour dire qu'elles ne sont plus parlées. Mais qui, dans ce cas, sont bien peu mortes. Car si le mort saisit le vif, il est mal mort. Et sans nul doute 
langue grecque et langue latine saisissent ici la française, au double sens d'une saisie et d'un saisissement. Sur elle, elles exercent l'emprise de langues non tout à fait autres, reliées entre elles par des rapports de généalogie. Dont la présence post mortem hante leur descendante, l'ombre des vieux mots se profilant toujours derrière leur sens familier. Toujours prête à resurgir en pleine lumière pour « désassocier la convention ${ }^{3}$ ». Pour frapper de saisissement; pour dessaisir des certitudes et des habitudes de pensée.

7 Portés en italique dans le cours du texte, comme le veut l'usage, ou typographiés en romain, les substantifs sarx et hiems surgissent de loin en loin, chacun dans son livre, pour faire trou dans la surface monolingue. Faire brusquement tomber du français dans une autre langue. Une de ces deux langues que l'on dit anciennes mais que Pascal Quignard préfère nommer aïeules, ou originaires, et dont l'hésitation typographique, caractéristique des deux livres, souligne encore le statut ambigu, entre éloignement et proximité. D'autant que les deux textes font un usage récurrent des termes "chaos", "trauma ", " hiatus ", passés sans modification de forme - mais non sans inflexion de sens - du grec ou du latin au français, et dont l'isomorphisme, en l'absence d'un respect constant des codes typographiques, impose une lecture vacillante, sans cesse trébuchant d'une langue à l'autre. Ou, pour le dire avec Pascal Quignard lui-même, contraint à « lire en deux langues ». Ce qui équivaut peut-être à n'en " lire aucune » par obligation de percevoir l'" intervalle physique et erratique ${ }^{4}$ » entre elles. Soit la phrase "Hommes ont les lèvres de sarx et de chaos» (p.145): l'uniformité typographique de l'ensemble qui, en refusant l'usage attendu de l'italique, refuse de rendre le grec sarx à son étrangeté linguistique déstabilise la lecture du substantif final. Chaos français ou chaos grec? Impossible de trancher. L'un et l'autre sans doute - d'où la nécessité de tenir ensemble tous les sens des deux langues. L'un et l'autre, et, plus encore, l'intervalle entre eux : le trou, précisément, qui s'ouvre; entre les langues et dans les langues; dans l'épaisseur sédimentée des langues ; nous laissant bouche bée, «lèvres » directement ouvertes sur l'abîme du temps creusé soudain au cœur de la langue.

L'épaisseur temporelle, rien ne la révèle mieux que l'étymologie qui, soucieuse d'exactitude ou portée par une rêverie mimologique, commande très souvent la progression de la pensée. Revenir au latin ou au grec, langues aïeules, pour ouvrir grâce à elles des brèches dans la langue française, c'est souvent en effet opérer une brusque remontée vers l'origine par le recours au processus étymologique, tantôt explicite dans le cas de Sarx, tantôt sous-jacent comme il l'est dans Hiems.

9 Rendu à son altérité par l'emploi de l'italique ou quasi-naturalisé par celui du romain ce qui le rend, paradoxalement, plus autre encore -, le mot sarx ponctue et conduit une méditation sur le sarcasme qui emprunte au moins en partie la forme d'une remontée $\mathrm{du}$ temps dans et par les langues. Sarx, par sarkazein donne sarkasmos, qui donne sarcasmus, qui donne «sarcasme ». Ainsi sous le français « sarcasme », le petit traité de Quignard fait entendre le latin sarcasmus, sous le latin sarcasmus le grec sarkasmos et dans le grec sarkasmos le grec sarx.

$10 \mathrm{Si}$, à la différence de ce qui se produit pour le passage de "sarcasme " à sarx, la remontée du substantif français " hiver » à son synonyme latin hiems, qui est aussi son origine, ne suppose pas le franchissement de toutes les étapes d'un processus de dérivation étymologique - $\mathrm{d}^{\prime}$ ' hiver » à hibernum; de ce dernier à hibernus dont il est la substantivation et d'hibernus à hiems sur lequel ce qualificatif latin est lui-même formé -, si elle s'opère par l'intermédiaire d'une définition et d'une traduction : 
L'hiver est le temps qui suit le solstice (le temps qui suit le solstice en latin se dit hiems $^{5}$.)

11 le mouvement qui consiste à peser sur le mot pour l'ouvrir à une antériorité reste le même. Il implique seulement le recours à une étymologie plus proche de la rêverie cratylienne que de la science linguistique, celle « que Varron donnait à hiems ", dans le livre VI de la Lingua latina:

[...] vel, quod tum anima quae flatus omnium apparet, ab hiatu hiems.

... peut-être l'hiver vient-il de l'ouverture de la bouche parce que dans l'hiver

l'haleine est apparente.

(peut-être hiems vient-il de hiatus ${ }^{6} .$. )

12 Le vieux mot auquel le processus étymologique, explicite (Sarx) ou sous-jacent (Hiems), a reconduit aimante lui-même d'autres mots pris dans ces vieilles langues. Plus souvent d'ailleurs par dérive dans le sens que dans le son. Hiatus appelle ainsi, par synonymie, chaos et trauma. Quant à sarx, il engage une incessante avancée de vocable en vocable, moins coulée que spasmée, «[b]refs, brusques accès nominaux contrastants ${ }^{7}$ ». Ou prétendument concertants; mais dans ce cas le concert, le plus souvent, déconcerte. Sarx que Quignard a soin de traduire : "Sarx : chair », se précise ainsi par antonymie déclarée: "Sarx: chair. À l'inverse. Corpus» (p.142) ou par rapprochement non explicité : «Physis, sarx. La terre // Sterquilinum. Sterculinum : sarcasmes » (p. 144). Basculant du grec au latin voire au français, l'écriture contraint, par l'abrupt même des formules, à s'interroger sur ce qui peut bien faire de corpus l'inverse de sarx; sur ce qui unit sarx à physis puis, en une sorte de chaîne (synonymique ?), au français «terre "; sur ce qui, de ce fait, l'oppose à " neige »: «Terre et sarx. // Non la neige » (p. 145). À aller, peut-être, pour élucider l'antonymie entre sarx et corpus exhumer l'équivalent latin du grec sarx: caro, carnis: la carne, la viande. Sarx, alors, le fragment et non la totalité ? la matière et pas la forme ? sans doute... mais surtout, la chose à mordre, à déchirer. Dans la méditation spasmodique de Quignard, où les mots des vieilles langues, volontiers rapprochés hors syntaxe, produisent des sortes de courts-circuits noétiques, sarx est plus chair que " chair »; plus physique, au sens plein du terme (« sarx : un logos de physis » est-il d'ailleurs noté, p. 146), parce que plus chargé d'animalité. Sarx ce n'est pas seulement la chair; c'est la chair offerte à la prédation d'une bouche ogresse. Préparée par l'énoncé "en grec, le verbe sarkazein: "mordre à la chair" ", la notice étymologique empruntée à Littré le confirme :

«De sarx, chair. Sarkasmos, sarcasmus : mordre à la chair". »

13 Par la vertu du «mot sarx» réactivé dans le français «sarcasme», ce substantif familier, d'abord triplement défini : «soit l'insulte adressée au corps de l'adversaire mort, soit l'ironie née de l'amertume, soit le dire-inverse» (p.140), se recharge d'une énergie violente que l'usage lui avait retirée. Le sarcasme, quittant le lieu immatériel du verbe et la généralité abstraite de la définition, prend force de geste. « Sarkasmos »la forme grecque - « ouvre la bouche à la chair, ouvre la chair déchirée de la bouche en deux lèvres pour déchirer la chair » (p.143). Déchirure double, donc. Celle qu'opère dans la face sarcastique l'écartement des lèvres formant les paroles d'insulte. Celle que produisent ces paroles dans le corps de l'autre. La puissance agressive rendue au sarcasme par la réactivation de l'étymon grec est telle que, dans le texte de Quignard, le sarcasme, "ce rire amer des deux lèvres déchirées sur leurs voix comme chairs » (p. 145), mord même son auteur. Mord la bouche qui veut mordre. 

et grecque conduit à la rencontre d'un lambeau décisif de texte grec. Lambeau, oui, plutôt que citation car les frontières au lieu d'être nettement, donc proprement, délimitées sont tremblées, déchirées, celles du texte originel ne recouvrant pas exactement celles de la très libre traduction française qui lui fait suite. Il s'agit d'un fragment étrangement référencé d'Hérodote (Enquête, IV, 649), où le voyageur présentant les usages des Scythes en vient à décrire le procédé en vigueur chez ce peuple guerrier pour scalper une tête - «écorch[er] sur le pourtour des oreilles » et « arrach[er] les peaux des visages des morts »-, avant de préciser la destination de ces dépouilles « dont pour la plupart, ils font des serviettes, mais certains des manteaux de berger ». La transformation des "peaux arrachées » en "serviettes » attachées à la bride du cheval suppose que «sur ces peaux écorchées, à l'aide d'une côte de bœuf, [soit] racl[ée] la chair. » Or, Quignard le relève, le verbe utilisé par l'écrivain grec pour dire cet écharnage, "ce verbe est sarkizein ». D'où un raccourci volontairement provocant qui vaut pour redéfinition du français sarcasme :

La serviette est le sarcasme. Peaux et chairs raclées des visages des morts ${ }^{10}$.

17 De l'insulte ou l'ironie à la "serviette", barbare et funèbre trophée, le mot «sarcasme» a, grâce au remuement dans ses profondeurs des vocables grecs sarkasmos, sarkazein, sarx - pris consistance matérielle. Il a, si l'on peut dire, pris figure, est lui-même devenu, selon un commentaire interne au texte, ce que «les grammairiens grecs, les grammairiens latins » appelaient « une figure-de-rhétorique ». Une image.

18 Et sans doute est-ce bien de cela qu'il s'agit : le mot grec, le mot latin, tels que Quignard les fait jaillir du mot français, font figure. Image. De même que le sarcasme est devenu, via le recours au grec, la serviette de peau humaine tannée, de même l'hiver, saison et mot, est rendu par le recours au latin à une sorte de concrétude: il s'identifie au «morceau de brume» (p.172) de l'haleine gelée, moins palpable peut-être que la serviette scythe mais non moins visible. Vocables anciens, bouts de vieilles langues, sarx et hiems sont, en somme, bien plus que des associations de sons et de sens : des noyaux ou des nœuds d'«images efficaces ${ }^{11} »$, pour le dire avec un traité plus tardif de Rhétorique spéculative.

19 Ce que Pascal Quignard fait, par l'intermédiaire des mots grecs et latins, resurgir de l'Antiquité, c'est donc moins tel ou tel trait de civilisation, telle ou telle réalité géographico-historique qu'une sorte de crudité rêvée, celle de temps plus proches de l'origine. Le mot ne tire pas après lui la chose : il ne nomme aucune des realia typiques de la civilisation latine ou de la civilisation grecque. Significativement, aucun des vieux mots qui trouent le texte français - corpus, mundus, deinon, physis, terror, quod (Sarx); ignis, grando, nix, glacies, pavor, stupor, turdus, logos, vestigium (Hiems) - ne désigne une réalité propre à la Grèce ou à la Rome ancienne, institution, monument, pièce vestimentaire, objet. Les mots-titres d'ailleurs le laissaient augurer : sarx, la chair proie offerte et lèvres écartées sur la parole sarcastique -; hiems, l'hiver et, inséparablement, le morceau de brume, le bout d'air visible flottant devant la bouche qui s'ouvre pour parler. De tels mots sont mots « de physis", mots physiques : mots de la nature et mots du corps. Ils ne disent rien ni de la Grèce ni de la Rome antique mais 
font signe vers une antériorité mal situable dans l'espace et dans le temps. Ils portent en eux - du moins Quignard le voudrait-il - un très ancien vestige.

Peut-être est-ce parce que l'Antiquité n'est ainsi, au temps de Sarx et Hiems, que le lieu d'une plus grande proximité avec la part obscure, archaïque, animale, pré-linguistique dont quelque chose peut subsister ou reparaitre dans ses langues, que le latin est dans les deux livres aussi bien médiéval et théologique que classique. Dans Sarx, une citation légèrement inexacte de l'Énéide que Ronsard déjà avait utilisée comme exemple de sarcasme : Italiam metire jacens ${ }^{12}$, et que Quignard reproduit comme il l'a trouvée dans le Littré, assortie du commentaire ronsardien, côtoie des fragments des Meditationes piissimae du Pseudo-Bernard. Quant à Hiems, il se tisse tout entier sur des lambeaux de la Summa theologiae de Thomas d'Aquin. Latin tardif, donc. Mais latin. Et, en cela seul qu'il est latin, antique. Archaïsme du français, propre à rendre une forme d'étrangeté à la langue maternelle, à l'ensauvager. Avec les citations de Sarx et Hiems, Quignard fait déjà pour le latin ce qu'il fera de façon systématique pour l'anglais dans L'Occupation américaine : il multiplie les passages dans la langue originale et les traduit aussitôt avec une volontaire gaucherie, s'en tenant à ce mot à mot rugueux à partir duquel il est d'usage d'élaborer une traduction en bon français lorsqu'on se livre à l'exercice scolaire de la version :

Sicut fumus « repraesentat » ignem et talis " repraesentatio » dicitur esse " repraesentatio vestigii ». Vestigium enim demonstrat mutum alicujus transeuntis sed non qualis sit. (« La fumée "représente" le feu et une telle "représentation" est dite "de vestige". Vestige montre mouvement-chose-passant ; ne dit pas quelle elle est $\left.{ }^{13}[. .] ».\right)$

D'une phrase à l'autre, la traduction de Thomas d'Aquin glisse à ce que Pascal Quignard appelle ailleurs du "petit latin", sorte de translation maladroite qui a pour effet d'accroître la rudesse de la langue maternelle, de la « reconfier [...] à la barbarie qui [la] hante ${ }^{14}$ ». Oui, dans le vacillement d'une langue à l'autre, si caractéristique des deux petits livres de 1977, et quelles que soient les modalités de celui-ci, le grec et le latin barbarisent le français, par un constant retournement des langues savantes en langues barbares. Ou à tout le moins en langues plus crues, plus cruelles. Paradoxe souvent noté par les critiques, la rudesse s'obtient par l'érudition même.

Par la suite, Quignard, sans cesser de chercher dans le grec et le latin de quoi bousculer le français et lui conférer une plus grande force concrète, pourra, en creusant les mots jusqu'à leurs racines, ramener du fond du temps des fragments de mondes morts. Avec Le Sexe et l'Effroi, un " mot romain difficile : fascinatio " traînera par exemple dans son sillage des vases grecs et des fresques romaines qui viendront tour à tour appuyer les citations et alimenter l'effort pour penser «la métamorphose de l'érotisme joyeux et précis des Grecs en méditation effrayée ${ }^{15}$ ». Pour l'heure, les vieux mots, nœuds d'«images efficaces", supportent une pensée non conceptuelle de la langue en sa violence. Chaos, hiatus écartent ainsi les lèvres de chair pour ouvrir dans la face un trou d'autant plus physique qu'il s'abouche à la béance même de la terre, à l'abîme primordial des cosmogonies. Ce faisant, chaos et hiatus, comme sarx et hiems, comme tous les autres vocables grecs et latins, contribuent puissamment au fonctionnement poétique de la langue dans deux livres où l'intention noétique s'accompagne du refus de tout raisonnement discursif, logiquement lié. Au rebours de l'usage qui les dit «mortes", les langues anciennes sont ici au service d'une langue de poésie, la plus vivante et la plus corrosive de toutes. 


\section{NOTES}

1. Ces propos de P. Quignard sont cités par V. LANDEL, «Le passé recomposé », Magazine littéraire 233 (septembre 1986), p. 56.

2. Sarx et Hiems ont été réédités dans P. QUIGNARD, Écrits de l'éphémère, Galilée, 2005, p. 137-151 et p. 165-180. C'est ici l'édition de référence. On peut également lire Hiems dans le livre anthologique d'E. HOCQUARD et R. LEVY, Orange Export Ltd., 1969-1986, Flammarion, 1986, p. 131-138.

3. Rhétorique spéculative, Calmann-Lévy, 1995, rééd. Gallimard, « Folio », 1997, p. 13.

4. « Langue », Petits traités, Maeght Éditeur, 1990, rééd. Gallimard, « Folio », 1997, I, p. 498.

5. P. 170.

6. P. 171.

7. « Le misologue », Petits traités I, p. 50.

8. P. 142.

9. Étrangement référencé car ni le nom de l'auteur ni le titre de l'ouvrage ne sont portés. Seuls apparaissent, entre parenthèses, les numéros de livre et de paragraphe : « (lib. IV, 64)».

10. P. 146.

11. «Fronton », Rhétorique spéculative, p. 40.

12. Dans l'Énéide (XII, 360), la formule sarcastique est la suivante : Hesperiam metire jacens (« tu mesures de ton cadavre l'Hespérie $")$.

13. Thomas d'Aquin, Summa theologiae, quest. XLV, art. 7 Utrum vestigium aliquod Trinitatis sit in rebus creatis ( Y Y a-t-il un vestige de la Trinité dans les êtres créés »); cité p. 175.

14. P. Quignard à C. LAPEYRE-DESMAISON, Pascal Quignard le solitaire, Les Flohic, 2001, p. 136.

15. Le Sexe et l'Effroi, Gallimard, 1994, p. 12.

\section{AUTEUR}

\section{CATHERINE SOULIER}

Université Paul-Valéry Montpellier III

catherine.soulier@wanadoo.fr 